\title{
Digitized two-parameter spectrometer for neutron-gamma mixed field
}

\author{
Zdenek Matej, Jiri Cvachovec, Vaclav Prenosil, Frantisek Cvachovec and Sergey Zaritski
}

\begin{abstract}
This paper shows the results of digital processing of output pulses from combined photon-neutron detector using a commercially available digitizer ACQUIRIS DP 210. The advantage of digital processing is reduction of the apparatus in weight and size, acceleration of measurement, and increased resistance to pile-up of pulses. The neutron and photon spectrum of radionuclide source ${ }^{252} \mathrm{Cf}$ is presented.
\end{abstract}

\section{INTRODUCTION}

$\mathbf{T}$ HE recent development in the area of digital data processing enables dramatic reduction of the spectrometric system; this is achieved by converting the time course of the detected pulse into numeric samples at the output of the digitizer. Further data transmission and processing can be done exclusively digitally. The above described procedure has a lot of advantages:

- Down-sizing of the spectrometric devices

- Increased measurement rate (i.e. a shorter and thus cheaper measurement is sufficient)

- Possibility of advanced pulse shape analysis - e.g. evaluation of superimposed impulses caused by fast sequence of particle detections.

- In certain cases, another characteristic of the output impulse can be determined - the characteristic duration (time constant) of the output pulses.

The last mentioned parameter sometimes carries very useful information about the nature of the detected particle. This applies e.g. to organic scintillator of type stilbene or NE-213. The distinguishing of nature of the particles (in our case neutron/photon) is based on the different relation of the probability of triplet state excitation in the molecule of the scintillator (the de-excitation generates the slow part of the fluorescence) to the linear stopping power of the detected charged particles. The neutron scintillation (a neutron is detected by means of a scattered proton) has a longer decay than the photon impulse (a photon is detected by means of a scattered electron). On the other hand, for proportional detectors filled with hydrogen the rise time of a neutron impulse is shorter than that of a photon

Manuscript received on 16/05/2011. The work presented in this paper has been supported in the frame of specific research of the Department of Mathematics and Physics, University of Defense Brno in 2010, specific research of the Faculty of Informatics, Masaryk University in 2010 and research project SPECTRUM, No. TA01011383 of the Technology Agency of the Czech Republic.

Z. Matej, Faculty of Informatics, Masaryk University, Botanicka 68a, 602 00 Brno, Czech Republic. E-mail: xmatejz@mail.muni.cz.

J. Cvachovec and V. Prenosil is with Masaryk University, Brno, Czech Republic.

F. Cvachovec is with University of Defence, Brno, Czech Republic.

S. Zaritski is with Kurchatov Institute, Moscow, Russia.

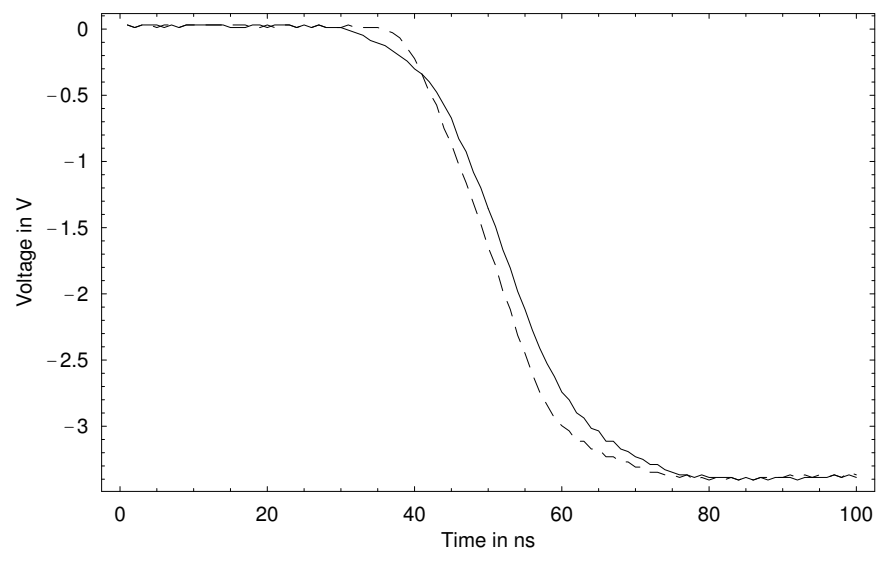

Fig. 1. Example time courses of neutron (solid) and photon (dashed) pulses.

impulse; this is again caused by different linear stopping power of the secondary charged particles (proton, electron). By adjusting the load resistance at the output of the detector, we can achieve a state where the speed of the increasing part of the output voltage impulse (rise time) reflects the differences in the time course of the current impulses. This different information carried by the time courses can be utilized to distinguish the neutron impulse from the photon one. The time courses of a neutron and photon pulse are shown in Fig. 1. In this case, the scintillation time constant is significantly smaller then the time constant of the circuit consisting of a working resistance and parasitic capacitance. We can see that a neutron pulse rises 2-3 ns longer than a photon pulse.

In the past, analog circuits were used to distinguish the courses. Since 2000, attempts have been made to evaluate both parameters (energy, type of particle) from the digitized output impulse. It is usual to arrange the measured data into a 3-D representation where one axis (e.g. $x)$ represents the rise time (type of the detected particle), another (y) stands for amplitude (energy), and the last one (z) records counts of impulses of the same parameters. This distribution, which we measured with ACQUIRIS DP 210 digitizer, is shown in Fig. 2. We can clearly distinguish neutrons and gamma part in this distribution. Moreover, other types of charged particles, noise, disturbances can be identified. Further processing leads to spectral energetic distribution.

\section{EXPERIMENTAL DATA PROCESSING}

The digitizer ACQUIRIS DP 210 converts the initial part of each input pulse (i.e. output signal from the detector) into 100 samples with a repetition frequency of 1 or $2 \mathrm{GHz}$. 




Fig. 2. 3-D distribution of output pulses from stilbene detector. The $\mathrm{x}$-axis represents pulse rise time, $y$-axis amplitude, and z-axis count of pulses of the same parameters corresponding to 5 and 95 percent of the amplitude. Gamma part on the left, neutron part on the right.

These 100 values (obtained as discrete data) represent the most important part of the pulse containing information on the amplitude (energy) and the timing of the rising part of the pulse (nature of the particle). The usually measured neutron spectra in the interval 0.5 to $10 \mathrm{MeV}$ require the recording of about one million pulses. Since the neutron pulses are sampled simultaneously with the photon ones without knowing the nature of the particle and the ratio of neutrons to photons in the mixed fields is more than ten, we receive relatively large amounts of data. The data is transported to memory, where it is stored for off-line processing because on-line processing cannot be performed quickly enough.

Further data processing is performed so that for each pulse (100 numbers) we find the largest number - amplitude - and then we determine the time interval $\tau$ between samples.

According to that $\tau$ we should ideally be able to divide all pulses into two groups - neutrons and photons. However, reality is more complex and the result is a distribution as shown in Fig. 2. It is obvious that the $\mathrm{n} / \mathrm{g}$ pulses can be easily distinguished for higher amplitudes but human interaction is still required for lower energies. This distribution is then split into neutron and photon parts; further, it is adjusted into a form required by the codes solving the Fredholm integral equation for the evaluation of the final product, i.e. the neutron and photon energy spectrum.

The processing of the pulses with the current digitizer is not ideal because of its low dynamic range of quantization ( 8 bits). For neutrons from energy interval 0.5 to $10 \mathrm{MeV}$, the ratio between the lowest and the highest pulse amplitudes is ca. 1:200. We solved this problem by taking the measurements at three different voltage levels for the photomultiplier; the resulting amplitude distributions have been merged by a software routine.

\section{THE EVALUATION OF THE ENERGY SPECTRUM}

When evaluating experimental data from the detector, we meet the linear inverse problem that can be formulated in case of neutron spectrum as follows: Let $g\left(E_{p}\right)$ and $A\left(E_{n}, E_{p}\right)$ be (continuous) functions. A (continuous) function $f\left(E_{n}\right)$ is to be found such that

$$
g\left(E_{p}\right)=\int A\left(E_{n}, E_{p}\right) f\left(E_{n}\right) d E_{n} .
$$

The above equation models the process of measurement using various devices or e.g. the output of a graphical device. It is a Fredholm integral equation of the first kind where

- $A\left(E_{n}, E_{p}\right)$ is the kernel, a characteristic of the measuring device, often called the response function. Its discrete approximation is determined by Monte Carlo modelling and measurement of monoenergetic neutron sources.

- $g\left(E_{p}\right)$ is the measured proton spectrum, i.e. experimental data. (Neutrons are detected by means of protons.)

- $f\left(E_{n}\right)$ is the result to be found, i.e. the neutron spectrum.

In general, equation (1) has no analytical solution and thus must be solved in a discrete form:

$$
\boldsymbol{g}=A \boldsymbol{f}
$$

where $\boldsymbol{g}=\left(g_{1}, \ldots, g_{m}\right)^{T}, A$ is a $m \times n$ matrix, and $\boldsymbol{f}=\left(f_{1}, \ldots, f_{n}\right)^{T}\left({ }^{T}\right.$ means transposition). For stilbene and NE-213 detectors $m=n$. Our options for stilbene are $m \approx 60,120,240$. We solve (2) by the maximum likelihood method [1]. It is a standard statistical tool for point estimations. For the maximizing of the likelihood function, we use a general iterative algorithm called Expectation Maximization, originally developed for image reconstruction in astronomy, medicine etc.

\section{Measurement Result And ENERgy SPECTRUm EVALUATION FOR NEUTRONS AND PHOTONS FROM THE ${ }^{252}$ CF SOURCE}

By the above described procedure we measured and evaluated neutron and photon spectrum of the ${ }^{252} \mathrm{Cf}$ source. The choice of this radiation source producing mixed field is not random. It is an artificial radionuclide and the neutrons originate from spontaneous fission of the $\mathrm{Cf}$ nuclei. Its neutron spectrum is widely regarded as standard and its shape can be used for testing correctness of the experimental method and accuracy of the evaluation process. It is very close to fission spectrum. Fig. 3 shows the neutron spectrum and Fig. 4 the photon spectrum of this source (measured with stilbene $45 \mathrm{x}$ $45 \mathrm{~mm})$.

\section{DisCUSSION}

The first measurement results of energy spectra show prospects of the experimental methods used and the possibility of further improvement. The methodology of discrimination of impulses belonging to different types of radiation (in our case, neutrons and photons) requires more precise mathematical methods for finding the characteristics in which the pulses of the two types of radiation differ. These can be various, e.g. Fourier analysis, the use of neural networks, machine learning etc. We have recently used the Support Vector Machines (SVM) technique. It is a machine learning method which seeks optimum classification of the training data into two categories 


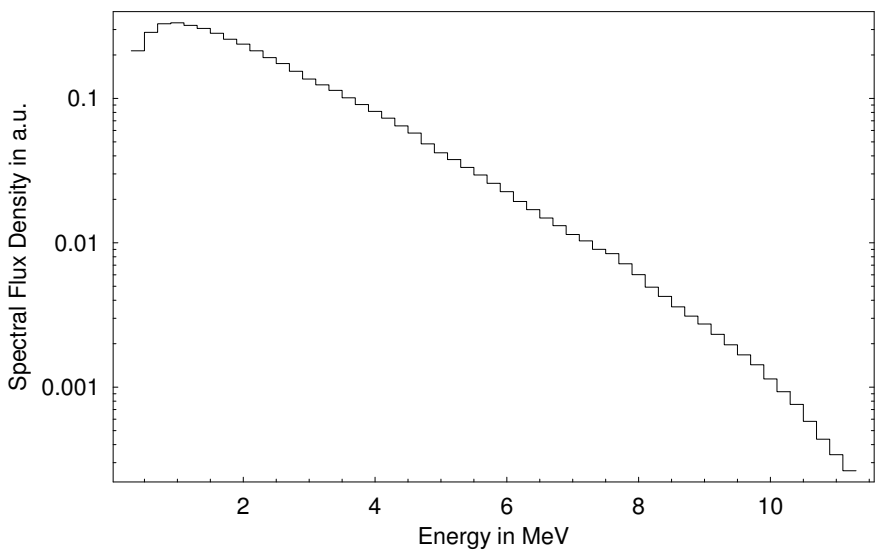

Fig. 3. Neutron spectrum $\varphi\left(E_{n}\right)$ from spontaneous fission of ${ }^{252} \mathrm{Cf}$.

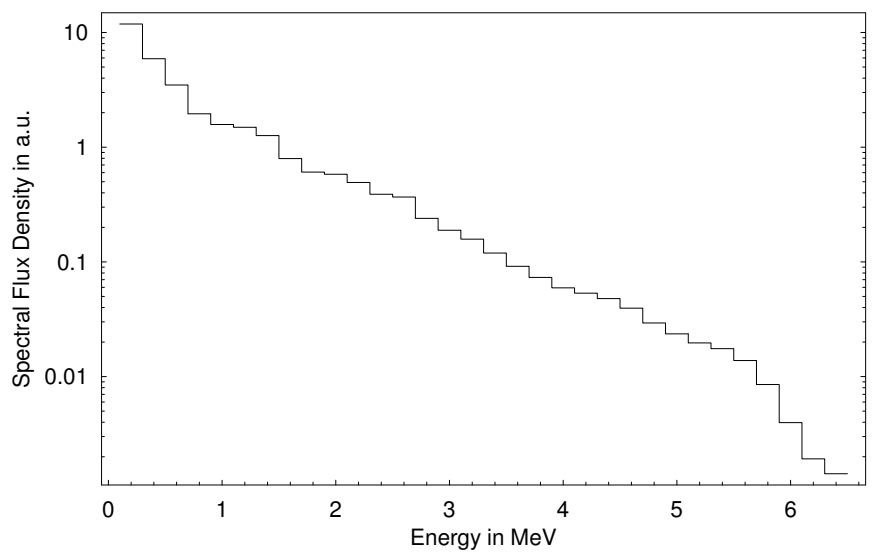

Fig. 4. Photon spectrum $\varphi\left(E_{n}\right)$ from spontaneous fission of ${ }^{252} \mathrm{Cf}$.

separated by a hyperplane. Simply said, this hyperplane maximizes the minimum distance between those two data sets. As SVM is a binary classifier, it can be used to distinguish neutron and proton pulses. Preliminary results, shown in Fig. 5, look very promising. Fig. 5(a) depicts training data representing well separated neutron and photon pulses corresponding to higher energy values. Fig. 5(b) shows the result of the SVM classification / separation. You can compare these results with Fig. 5(c) that illustrates the classical separation according to pulse rise time (e.g. between 5 and 95 percent of the amplitude).

Our device used for sampling the pulses is currently not high-end. The main problem is its small dynamic range (8-bit quantization), which forced us to measure the spectra in more steps and then combine the resulting parts in a complicated way. We want to achieve substantial improvements in our results in the above outlined areas.

\section{REFERENCES}

[1] J. Cvachovec, F. Cvachovec, Maximum likelihood estimation of a neutron spectrum and associated uncertainties, Advances in Military Technology, 2008, vol. 1, no. 2, p.67-79.

[2] F. Cvachovec, J. Cvachovec, P. Tajovsky, Neutron response function for stilbene detector in energy range 0.5 to $20 \mathrm{MeV}$, International Workshop on Neutron Field Spectrometry in Science, Technology and Radiation Protection (NEUSPEC 2000), June 04-08, 2000, Pisa, Italy.
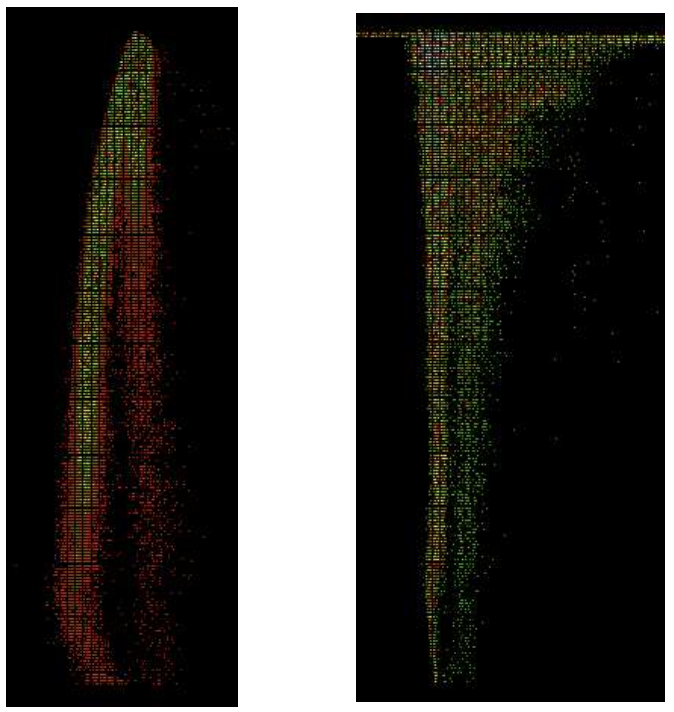

(a)

(b)

(c)

Fig. 5. (a) Training data, (b) Result of SVM separation, (c) Classical approach based on pulse rise time 